\title{
Modeling relative frost weathering rates at geomorphic scales
}

\author{
Alan W. Rempel ${ }^{\mathrm{a}, *}$, Jill A. Marshall ${ }^{\mathrm{b}, \mathrm{c}}$, Josh J. Roering ${ }^{\mathrm{a}}$ \\ ${ }^{a}$ Department of Geological Sciences, University of Oregon, Eugene, USA \\ ${ }^{b}$ Department of Earth and Planetary Science, University of California, Berkeley, USA \\ ${ }^{c}$ Institute of Arctic and Alpine Research, University of Colorado, Boulder, USA
}

\begin{abstract}
Frost damage is a powerful agent of geomorphic change. Cracks can grow when the ice pressure in pores reaches a threshold that depends on matrix properties and crack geometry. Mineral surfaces that are preferentially wetted by liquid water rather than ice are coated by premelted liquid at a pressure that is lower than the ice pressure. Because this pressure difference increases as the temperature cools, when the ice pressure is effectively pinned at the cracking threshold, temperature gradients induce gradients in liquid pressure that draw water towards colder temperatures. Porosity increases and frost damage accumulates in regions where water supplies crack growth. To apply this understanding over the large spatial and temporal scales that are relevant to evolving landscapes, we develop a simple model that tracks porosity changes. Our central assumption is that frost damage is correlated with porosity increases under conditions where frost cracking takes place. Accordingly, we account for the permeability reductions with decreased temperature that accompany ice growth along porous pathways and derive general expressions for the porosity change through time at particular depths, as well as the total porosity increase through all depths beneath a point at the ground surface over the time during which cracking occurs each year. To illustrate the resulting patterns of frost weathering, we consider a general case in which the permeability has a power law dependence on temperature and the annual surface-temperature variation is sinusoidal. We find that the degree of frost damage generally decreases with depth, except at localized depths where damage is elevated because the rock spends longer times near the threshold for cracking, leading to enhanced water supply in comparison with neighboring regions. The magnitude of the net expansion that results from porosity changes at all depths beneath the ground surface is
\end{abstract}

\footnotetext{
${ }^{*}$ Corresponding author

Email address: rempel@uoregon.edu (Alan W. Rempel)
} 
increased for seasonal thermal cycles with larger amplitudes, with a broad maximum centered on a mean annual temperature near the threshold required for crack growth. Warmer mean annual temperatures lead to less damage because of the reduction in time during which it is cold enough for cracking, whereas colder mean annual temperatures are accompanied by reduced water supply due to the temperature dependence of permeability. All of the controlling parameters in our model are tied explicitly to physical properties that can in principle be measured independently, which suggests promise for informing geomorphic interpretations of the role of frost weathering in evolving landforms and determining erosion rates.

Keywords: periglacial processes, frost weathering, frost cracking, premelting, erosion, freeze-thaw

\section{Introduction}

Ice formation is widely recognized as a leading agent of physical weathering in nature (e.g. French, 2013; Hall, 2004; McGreevy and Whalley, 1982; Ollier et al., 1984) and costly deterioration to the built environment (e.g. Coussy, 2005; Ho and Gough, 2006; Scherer, 1999). Such frost damage takes place when the pressure exerted against pore walls exceeds the cohesive strength of moist porous media and causes cracks to extend. The density change upon solidifying liquid water (density $\rho_{l}$ ) into ice (density $\rho_{i} \approx 0.9 \rho_{l}$ ) can cause significant pressure increases (e.g. Davidson and Nye, 1985). However, the effectiveness of this particular mechanism in isolation is limited because the density change drives water flow away from the solidification front into unfrozen or unsaturated pore space, which precludes the generation of stress concentrations sufficient to propagate cracks (Hallet et al., 1991). Instead, the efficacy of frost damage stems from the common tendency for liquid to flow in the opposite direction and supply crystal growth so that the mass of ice in the frozen pore space exceeds the mass of water that was present initially at warmer temperatures (e.g. Akagawa and Fukuda, 1991; Taber, 1930). (A particularly convincing demonstration of this physical mechanism was first revealed by Taber (1930) through experiments that demonstrated "frost" expansion during the freezing of two liquids, benzene and nitro-benzene, that have higher densities in their solid states.) This flow is a consequence of the phase behavior, referred to as interfacial premelting, that induces liquid water to wet interfaces between the pore walls and ice at temperatures $T$ that are colder than the normal bulk melting temperature $T_{m}$ (e.g. Dash et al., 2006; Wettlaufer and Worster, 2006). Changes in the strength of the interfacial forces that cause this wetting behavior produce liquid pressure gradients that commonly are aligned with gradients 
in temperature. These liquid pressure gradients drive the redistribution of water mass that is a defining characteristic of the range of phenomena that result from segregation ice growth, which has enjoyed a particularly long history of study in the context of frost heave (e.g., Rempel, 2010). More recently, this mechanism has been invoked to explain spatial and temporal variations in erosion rate (e.g. Delunel et al., 2010; Hales and Roering, 2005; Marshall et al., 2015), yet the basis for relating the physics of this process to geomorphic work requires justification. Here, we consider segregation ice growth in cohesive porous media and present a mechanistic description that is amenable for application over the large spatial and temporal scales relevant for understanding the role of frost weathering in landscape evolution.

While the detailed thermo-mechanical interactions that produce frost damage can be influenced by the density change upon freezing and ubiquitous dissolved salts, the essential behavior can be understood without these complicating factors. The equilibrium presence of liquid water below $T_{m}$ (i.e. $273 \mathrm{~K}$ ) is accompanied by a difference between the ice and liquid pressures (Dash et al. 2006). As $T$ drops so that the undercooling $\Delta T \equiv T_{m}-T$ increases, there is a proportional increase in the strength of the intermolecular forces that both give rise to this pressure difference and enable ice to exert pressure against the pore walls across the intervening premelted liquid (Dash, 1989; Rempel et al., 2001). The total force exerted on the solid matrix must be balanced and the resistance to deformation is inevitably characterized by stress concentrations near the tips of crack-like regions where the pore surface curvature is high. Frost damage occurs when conditions are not only sufficiently cold that the net pressure exerted against the pore walls pushes the stress concentrations to levels where the matrix material fails and fractures, but also warm enough to enable the liquid flow that supplies ice growth into the newly opened space (Hallet, 2006; Walder and Hallet, 1985). The range of temperatures that meet these criteria has been used to define a "frost-cracking window" within which most damage takes place (Anderson, 1998).

The mechanics that control frost damage in idealized systems have been examined in considerable detail using both theoretical and experimental approaches (e.g. Murton et al., 2006; Vlahou and Worster, 2015; Walder and Hallet, 1985). Some of the resulting insights have been incorporated within several parameterized measures that are designed to gauge differences between the relative intensity of frost damage expected at the much larger scales relevant to geomorphic change. The simplest of these formulations associates relative frost damage with the total time spent within a frost-cracking temperature window (e.g. $-8^{\circ} \mathrm{C}<T<-3^{\circ} \mathrm{C}$, see Anderson, 1998). Noting the 
direct relationship between temperature gradients and the pressure gradients that drive the liquid flow needed to supply ice growth in cracks, a second measure of the tendency for damage weights the time within the frost-cracking window by the temperature gradient, while also requiring the presence of a flow path along which the temperature cools monotonically from positive values (Delunel et al., 2010; Hales and Roering, 2007, 2009; Marshall et al., 2015; Savi et al., 2015). A variant on this approach prioritizes hydraulic resistance by deploying the distance between an unfrozen liquid source and the potential frost-cracking location within an exponential penalty function that modifies the gradient-weighted time integral (Anderson et al., 2013; Scherler, 2014); the weighting function has also been augmented to include a dependence upon the volume of liquid present along connected flow paths (Andersen et al., 2015). The patterns of frost damage predicted by these methods share common features, but there are also significant differences. Moreover, it is not obvious how changes to some of the basic physical parameters that are known to control frost damage in idealized systems can best be represented. Although it is not practical to detail the evolution of each crack-like pore that is subject to freezing conditions within a given landscape, improved confidence in predictions for the patterns of frost damage through space and time can be gained by revisiting and building upon the mechanical understanding achieved in studies of the incremental damage that accumulates in simple systems. The study of crack growth in bergschrunds by Sanders et al. (2012) is notable for taking the direct approach of tracking crack extension at a discrete series of depths and developing a frost damage index based upon an ensemble average of outcomes from calculations that sample a distribution of controlling parameters. Here, we take a continuum approach and retain the computational simplicity of earlier qualitative damage indices that do not explicitly track the evolution of discrete cracks; instead, we associate the propensity for damage with the accumulation of ice that increases porosity in locations were cracks can grow.

In discussing the implications of earlier field and laboratory work, McGreevy and Whalley (1982) suggested that "it would seem reasonable to assume that for most rocks the degree of frost damage can be equated with the amount of ice (that) forms in them during freezing". The mechanical interactions that determine the degree to which ice-filled pores can expand and produce frost damage are now understood well enough to make quantitative predictions for ice accumulation through space and time. Because the permeability to liquid flow decreases sharply with temperature in frozen porous media, the total water flux along a flow path largely driven by the temperature gradient must drop as the temperature decreases; mass conservation ensures that porosity increases as a result 
and frost damage accumulates provided that the ice exerts sufficient stress in the porous medium to propagate cracks. In essence, rather than explicitly tracking crack growth, the generation of porosity is treated here as a measure of frost damage induced by segregation ice. We show how the measurable parameters that characterize a given setting (including rock properties) and thermal history combine to determine the spatial and temporal patterns of damage that are implied by this connection. To forecast the degree of frost damage at larger scales, we show how the annual temperature variation at the ground surface can be combined with considerations of heat and mass transport to quantify the ice-induced expansion of porous materials during the portion of the year in which frost-cracking is active beneath a particular location. This enables an estimate of the potential for frost damage that is tied explicitly to the increase in water mass that accompanies ice formation

\section{Porosity changes during freezing}

The basic premise underlying our treatment is that the frost damage caused by crack growth correlates with increases in porosity that are made possible through water supply to growing ice. Any valid description of these processes must account for the controlling thermo-mechanical properties and phase behavior, as well as the evolving environmental conditions. In this section, we begin by outlining the simplest set of assumptions that is needed to develop such a model. We then explore the model predictions that follow, before moving on to discuss some of their implications for geomorphic change and the complications that can arise from the incorporation of additional features that we neglect in our initial treatment.

\subsection{Model development}

We proceed from the following set of assumptions (elaborated upon in the Supplementary Information): i) rock strength limits the region where frost damage takes place to locations where the ice pressure matches a characteristic value $P_{c}$ (e.g. Vlahou and Worster, 2015; Walder and Hallet, 1985) - all else being equal, $P_{c}$ scales directly with fracture toughness and inversely with the square root of crack size; ii) the pressure difference $\Delta P$ between the ice and liquid phases is set by the generalized Clapeyron equation to be proportional to the undercooling $\Delta T=T_{m}-T$ so that $\Delta P \approx\left(\rho L / T_{m}\right) \Delta T$, where $\rho L$ is the volumetric latent heat and the ratio $\rho L / T_{m} \approx 1.1 \mathrm{MPa} / \mathrm{K}$

(e.g. Dash et al., 2006; Wettlaufer and Worster, 2006); iii) the liquid flux satisfies Darcy's law with 
a temperature-dependent permeability $k(T)$ that accounts for the progressive clogging of liquid pathways that accompanies ice formation (e.g. Andersland and Ladanyi, 2004; Walder and Hallet, 1985 ); iv) the model domain is completely water-saturated (i.e. pore volume $=$ ice volume + liquid volume); and v) ongoing ice growth is slow at temperatures cold enough for frost damage to take place, so latent heat is unimportant under these circumstances and the temperature evolution satisfies the heat equation (i.e. $\partial T / \partial t=\kappa \nabla^{2} T$ ) with uniform thermal diffusivity $\kappa$.

Together, the first and second conditions imply that the upper temperature limit at which frost damage can take place corresponds with an undercooling of

$$
\Delta T_{c} \approx \frac{T_{m}}{\rho L}\left(P_{c}-P\right) \approx \frac{T_{m}}{\rho L} P_{c},
$$

where the second approximation is valid as long as the water pressure $P$ outside the region where active frost-cracking takes place remains close to ambient levels (i.e. $10^{5} \mathrm{~Pa}$ ). In practice, values of $P_{c}$ in the range of a few MPa are typically needed to propagate mm-cm scale cracks (see Atkinson, 1984, for an extensive review of fracture toughness in geological materials); such levels are much greater than fluid pressure variations encountered in most systems of interest. Consistent with empirical observations of the upper temperature limit on the frost-cracking window, common values of $\Delta T_{c}$ predicted by equation (1) are on the order of a few degrees centigrade (i.e., $-\Delta T_{c} \approx-2$ to $\left.-6{ }^{\circ} \mathrm{C}\right)$.

For the common case where heat flow is in the direction normal to the ground surface, the remaining numbered conditions above combine with mass balance considerations to imply that the change in porosity $\Delta n$ at depth $z$ from the time $t_{c}$ that a particular point cools to reach $\Delta T_{c}$ until some later time $t$ is (see the Supplementary Information)

$$
\Delta n(z, t)=\frac{\rho L}{T_{m} \mu}\left[\int_{t_{c}}^{t} \frac{\mathrm{d} k}{\mathrm{~d} T}\left(\frac{\partial T}{\partial z}\right)^{2} \mathrm{~d} t+\kappa^{-1} \int_{T_{m}-\Delta T_{c}}^{T_{m}-\Delta T} k \mathrm{~d} T\right],
$$

where $\mu$ is the liquid viscosity. The first integral on the right describes the accumulation of water caused by temperature-dependent changes in the permeability while the second integral accounts for the effects of changes in the liquid pressure gradient at a given point over time. Integrating this expression over an annual cycle during which the temperature is assumed to return to its initial value so that the upper and lower limits on the second integral are identical, the change in porosity that is accompanied by frost damage at a particular location is

$$
\Delta n(z)=\frac{\rho L}{T_{m} \mu} \int_{\Delta T>\Delta T_{c}, 1 \text { year }} \frac{\mathrm{d} k}{\mathrm{~d} T}\left(\frac{\partial T}{\partial z}\right)^{2} \mathrm{~d} t,
$$


where the integration limits represent the portion of a single year during which $\Delta T>\Delta T_{c}$. Note that as long as permeability decreases monotonically with decreased temperature, as is generally expected from the progressive clogging of flow pathways by ice formation, equation (3) predicts net porosity always increases during the portion of the year spent under frost-cracking conditions. This is a significant point of departure from previous treatments that have required a connecting flow path with regions where $T>T_{m}$ (e.g. Andersen et al., 2015; Anderson et al., 2013; Hales and Roering, 2007); as explained further in the Discussion, we expect liquid supply from warmer domains to continue even if they are colder than $T_{m}$.

To gauge the degree of frost damage beneath a particular location on the ground surface (or within a particular rock unit), $\Delta n(z)$ can be integrated over depth to obtain the added pore volume per unit cross-sectional area during the portion of a year in which $\Delta T \geq \Delta T_{c}$ as

$$
\lambda=\frac{\rho L}{T_{m} \mu} \int_{1 \text { year }}\left(\left.k \frac{\partial T}{\partial z}\right|_{z^{+}, \Delta T \geq \Delta T_{c}}-\left.k \frac{\partial T}{\partial z}\right|_{z^{-}, \Delta T \geq \Delta T_{c}}\right) \mathrm{d} t
$$

$\lambda$ is equivalent to the total expansion that results from ice formation beneath a given point. The integrand in equation (4) accounts for water flow into the frost-cracking domain from above (first term) and below (second term) as the difference between the product of the permeability and temperature gradient evaluated at the moving upper $z^{+}$and lower $z^{-}$boundaries of the region with $\Delta T \geq \Delta T_{c}$. This formulation neglects the potential for the near-surface water content to diminish over long time periods in permafrost conditions, as outlined further in the Discussion.

\subsection{Model predictions}

With equation (1) setting the upper temperature limit required for frost cracking, when the porosity change caused by ice formation correlates with frost damage equation (2) provides a measure of the evolution of frost damage at a particular depth, equation (3) describes the cumulative damage at a particular depth through the course of a year, and equation (4) gauges the total damage accumulated in a year at all depths beneath a particular location on the ground surface. Alongside the physical constants appearing in these equations, the temperature-dependent permeability and the thermal field itself must still be specified in order to quantify the expected patterns of damage that result.

Measurements and theoretical descriptions of the changes in permeability at sub-zero temperatures have been fit with a variety of functional forms (e.g. Kurylyk and Watanabe, 2013; Lebeau 
and Konrad, 2012; Watanabe and Flury, 2008), but a simple power-law approximation is commonly adequate (validated in fine-grained soils, e.g. Andersland and Ladanyi, 2004)and will be adopted here to illustrate the basic controls on frost damage. Taking $k_{0}$ as the permeability in ice-free regions and $\Delta T_{f}=T_{m}-T_{f}$ as the undercooling at which ice first appears in the pore space, for $\Delta T \geq \Delta T_{f}$ the permeability is written as

$$
k=k_{0}\left(\frac{\Delta T_{f}}{\Delta T}\right)^{\alpha},
$$

where the empirical exponent $\alpha$ is typically around 4 . The threshold undercooling for ice formation in pores is controlled by curvature effects so that $\Delta T_{f}=2 \gamma T_{m} /\left(\rho L R_{p}\right)$ where $\gamma \approx 0.035 \mathrm{~J} / \mathrm{m}^{2}$ is the ice-liquid surface energy, $R_{p}$ is a characteristic pore dimension, and the coefficient $2 \gamma T_{m} /(\rho L) \approx$ $0.06^{\circ} \mathrm{C} / \mu \mathrm{m}$. Note that this can also be written as $\Delta T_{f}=2 \gamma /\left(R_{p} P_{c}\right) \Delta T_{c}$, and since typically $P_{c} \gg 2 \gamma / R_{p}$ the permeability tends to decrease fairly dramatically with temperature changes of the scale $\Delta T_{c}$ that characterizes the regime in which frost cracking takes place. In a departure from most previous models, our treatment contains no explicit low-temperature cut-off for frost damage. Instead, the large reduction in permeability at colder temperatures stems the water supply needed for segregation ice growth and effectively sets the lower limit on the temperature range over which significant frost damage takes place.

This specific model for the thermal controls on permeability implies that equations (2) through (4) can be written as

$$
\begin{aligned}
\Delta n(z, t) & =\frac{D}{\Delta T_{c}^{2}} \int_{t_{c}}^{t}\left(\frac{\Delta T_{c}}{\Delta T}\right)^{\alpha+1}\left(\frac{\partial T}{\partial z}\right)^{2} \mathrm{~d} t+\frac{D}{\kappa} \frac{\left(\Delta T_{c} / \Delta T\right)^{\alpha-1}-1}{\alpha(\alpha-1)}, \\
\Delta n(z) & =\frac{D}{\Delta T_{c}^{2}} \int_{\Delta T>\Delta T_{c}, 1 \text { year }}\left(\frac{\Delta T_{c}}{\Delta T}\right)^{\alpha+1}\left(\frac{\partial T}{\partial z}\right)^{2} \mathrm{~d} t, \text { and } \\
\lambda & =\frac{D}{\Delta T_{c}} \int_{1 \text { year }}\left[\left.\left(\frac{\Delta T_{c}}{\Delta T}\right)^{\alpha} \frac{\partial T}{\partial z}\right|_{z^{+}, \Delta T \geq \Delta T_{c}}-\left.\left(\frac{\Delta T_{c}}{\Delta T}\right)^{\alpha} \frac{\partial T}{\partial z}\right|_{z^{-}, \Delta T \geq \Delta T_{c}}\right] \mathrm{d} t .
\end{aligned}
$$

where the diffusivity $D=\alpha \rho L k_{c} \Delta T_{c} /\left(T_{m} \mu\right)$ is a measure of the propensity for frost-induced porosity changes. Abrupt contrasts in $D$ are expected to be common at lithologic boundaries that are characterized by transitions in the rock properties and in the magnitude of frost damage, though the spatial and temporal patterns of damage may remain similar. Values for the physical constants $\rho, L, T_{m}$ and $\mu$ that define water properties are summarized near the top of Table 1 ; this leaves the threshold undercooling $\Delta T_{c}$, the corresponding permeability $k_{c} \equiv k_{0}\left(\Delta T_{f} / \Delta T_{c}\right)^{\alpha}$, and $\alpha$ as the primary variables controlling differences between $D$ in different materials. The rock strength 


\begin{tabular}{|lr|c|c|}
\hline parameter & & nominal value & typical range \\
\hline ice density $\left[\mathrm{kg} / \mathrm{m}^{3}\right]:$ & $\rho$ & 920 & n.a. \\
latent heat $[\mathrm{kJ} / \mathrm{kg}]:$ & $L$ & 334 & n.a. \\
bulk melting temperature $[\mathrm{K}]:$ & $T_{m}$ & 273 & n.a. \\
water viscosity $[\mathrm{mPa} \mathrm{s}]:$ & $\mu$ & 1.8 & n.a. \\
period [years]: & $P_{y}$ & 1 & n.a. \\
${ }^{a}$ thermal diffusivity $\left[\mathrm{mm}^{2} / \mathrm{s}\right]:$ & $\kappa$ & 1 & $0.1-2$ \\
${ }^{b}$ cracking pressure $[\mathrm{MPa}]:$ & $P_{c}$ & 3.3 & $1.8-5.5$ \\
${ }^{c}$ unfrozen permeability $\left[\mathrm{m}^{2}\right]:$ & $k_{0}$ & $10^{-14}$ & $10^{-18}-10^{-12}$ \\
permeability at $\Delta T_{c}\left[\mathrm{~m}^{2}\right]:$ & $k_{c}$ & $10^{-20}$ & $10^{-24}-10^{-16}$ \\
${ }^{d}$ power-law exponent []$:$ & $\alpha$ & 4 & $2-5$ \\
undercooling for cracking $\left[{ }^{\circ} \mathrm{C}\right]:$ & $\Delta T_{c}$ & 3 & $2-6$ \\
${ }^{d}$ undercooling for ice formation $\left[{ }^{\circ} \mathrm{C}\right]:$ & $\Delta T_{f}$ & 0.1 & $0.01-1$ \\
mean annual temperature $\left[{ }^{\circ} \mathrm{C}\right]:$ & $M A T$ & -5 & $-15-5$ \\
thermal amplitude $\left[{ }^{\circ} \mathrm{C}\right]:$ & $A$ & 10 & $5-15$ \\
diffusivity $\left[\mathrm{mm}{ }^{2} / \mathrm{s}\right]:$ & $D$ & $9.1 \times 10^{-5}$ & $10^{-9}-1$ \\
\hline
\end{tabular}

Table 1: Nominal parameter values used in model calculations and ranges of values that are considered typical from: ${ }^{a}$ tabulated thermal properties (Clauser and Huenges, 1995; Robertson, 1988); ${ }^{b}$ tabulated critical stress intensities (Atkinson, 1984) assuming cm-scale cracks; ${ }^{c}$ approximate unfrozen permeability for nominal pore size of $10^{-6} \mathrm{~m}$, with range estimated for pore sizes from two orders of magnitude smaller to one order of magnitude larger; ${ }^{d}$ based on frozen soils data compiled by Andersland and Ladanyi (2004). Other parameters with quoted ranges are derived following equations given in the text.

determines how large the undercooling must become before cracking can take place, thereby defining the integration limits through this control on $\Delta T_{c}$. The environmental conditions determine the integrands in equations (6)-(8), together with the exponent $\alpha$ that characterizes the variation in permeability with temperature.

To illustrate, we adopt a conductive sinusoidal temperature profile with mean annual temperature $M A T$, surface amplitude $A$ and period $P_{y}=1$ year, so that

$$
T(z, t)=M A T-A \exp \left(-z \sqrt{\frac{\pi}{\kappa P_{y}}}\right) \cos \left(\frac{2 \pi t}{P_{y}}-z \sqrt{\frac{\pi}{\kappa P_{y}}}\right),
$$

implying that the minimum surface temperature is attained at $t=n P_{y}$ for integer multiples $n$. 
More complex annual temperature variations, diurnal changes (neglected for now), contributions from latent heat at temperatures near $T_{f}$, changes in properties (e.g. $\kappa$ ) with depth, and the effects of snow-cover can all be important for contributing to the detailed thermal histories at different sites (e.g. Anderson et al., 2013; Marshall et al., 2015). However, the simple form of equation (9) is useful for highlighting the most basic controls on frost damage, independent of these additional complications.

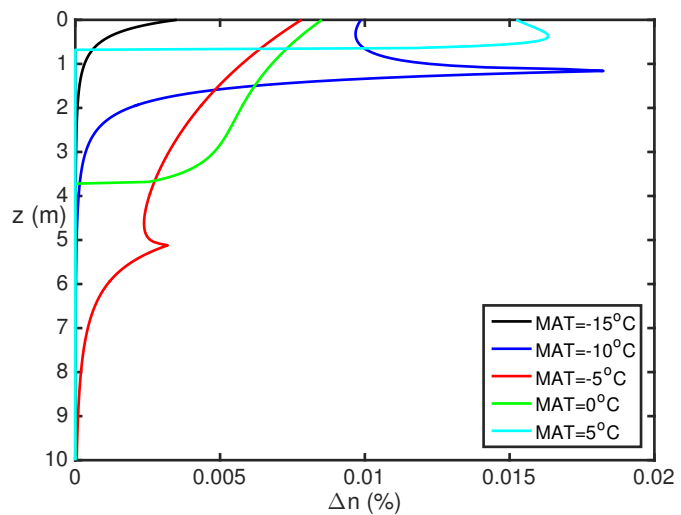

Figure 1: Porosity change $\Delta n$ from equation (7), shown as a function of depth for the mean annual temperatures indicated in the legend, using the temperature profile described by equation (9) with $A=10^{\circ} \mathrm{C}$ and the other nominal parameters listed in Table 1.

Figure 1 shows profiles of the increase in porosity $\Delta n$ predicted by equation (7) over the portion of a single year during which frost-cracking takes place, with each line showing the results for a different MAT; all other parameters are set to the nominal values listed in Table 1 . The depth range over which frost damage is expected corresponds with the range over which the undercooling surpasses $\Delta T_{c}$ (i.e., $T<-\Delta T_{c}$ ). For example, the undercooling reaches $\Delta T_{c}=3{ }^{\circ} \mathrm{C}$ everywhere from the ground surface down to nearly $4 \mathrm{~m}$ depth when $M A T=0{ }^{\circ} \mathrm{C}$, but only just over $0.5 \mathrm{~m}$ depth when $M A T=5^{\circ} \mathrm{C}$. In all the cases shown with $M A T<-\Delta T_{c}$, sufficiently low temperatures are reached for frost-cracking to take place at all depths. However, the drastic reduction in permeability at lower temperatures ensures that the largest changes in $\Delta n$ take place where $\Delta T$ spends the most time only slightly greater than $\Delta T_{c}$ (i.e., $T$ is slightly colder than $-\Delta T_{c}$ ). This flow requirement introduces considerable complexity to the form of the predicted $\Delta n$ profiles, for example leading 
to local maxima centered on particular depths (i.e. near $1 \mathrm{~m}$ for $M A T=-10^{\circ} \mathrm{C}$ and $5 \mathrm{~m}$ for $M A T=-5^{\circ} \mathrm{C}$ ) and a non-monotonic relationship between $M A T$ and $\Delta n$ at a particular depth. Lower down, the temperature approaches $M A T$ and $\Delta n$ tends to decrease with depth, in part because the temperature gradient is muted and in part because either the undercooling is not conducive to liquid flow (i.e. the permeability is too low) or it is not conducive to cracking (i.e. the ice pressure is too low).

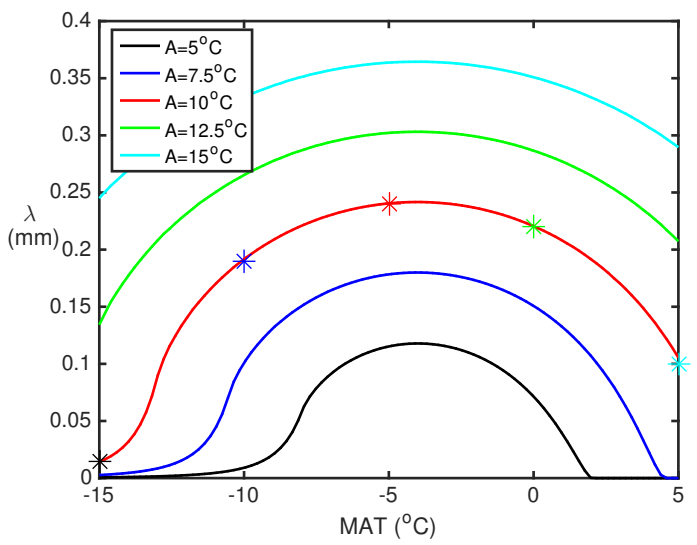

Figure 2: The integrated porosity change $\lambda$ from equation (8), shown as a function of mean annual temperature for the different thermal amplitudes indicated in the legend (i.e. ranging from $A=5{ }^{\circ} \mathrm{C}$ for the bottom line to $A=15^{\circ} \mathrm{C}$ for the line on top) and the temperature profile described by equation (9), using the nominal values for all other parameters listed in Table 1. The values of $\lambda$ for each of the profiles shown in Figure 1 correspond with the colored stars along the middle (red) line.

Figure 2 shows the net expansion $\lambda$ predicted by equation (8). All else being equal, $\lambda$ increases with the amplitude of the seasonal thermal cycle because of the direct dependence of the temperature gradient on $A$; higher gradients produce more dramatic permeability changes and drive rapid liquid transport. For low seasonal amplitudes (i.e. the lowermost two lines), no porosity changes are expected until $M A T$ is sufficiently cold that the undercooling surpasses $\Delta T_{c}\left(3^{\circ} \mathrm{C}\right.$ here $)$ in midwinter. As $M A T$ decreases further, the total seasonally and depth-integrated porosity change is expected to increase at first, as frost-cracking conditions become more pervasive. Once $M A T$ decreases sufficiently, however, the effects of the reduction in permeability with decreased temperature become important, particularly at times of the year when the undercooling throughout the entire 


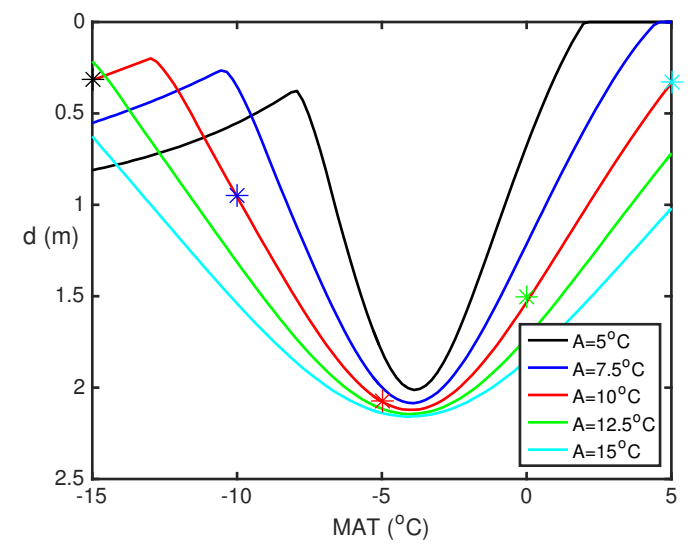

Figure 3: Swelling depth $d$ (note the reversed axis) at which the annual expansion during frost cracking reaches half its total thickness $\lambda$, shown as a function of $M A T$ for the different values of $A$ noted in the legend, and the other nominal parameters listed in Table 1. Stars highlight values of $d$ derived from the $\Delta n$ profiles shown in Figure 1.

rock column exceeds $\Delta T_{c}$. Although $\lambda$ tends to zero for very low $M A T$, the curves are asymmetric; the threshold undercooling $\Delta T_{c}$ for the onset of cracking has no corresponding low-temperature cut-off, with the effective lower bound on the "frost-cracking window" imposed instead by severe restrictions on permeability at lower temperatures.

The depth profiles for $\Delta n$ shown in Figure 1 are sufficiently different that their characterization in terms of $\lambda$ is not adequate for describing how the expected patterns of frost damage vary with the environmental forcing. A second useful measure is defined as the swelling depth $d$ over which half the total expansion is accommodated (i.e. $\int_{0}^{d} \Delta n(z) \mathrm{d} z=\lambda / 2$ ). Figure 3 shows how $d$ varies with $M A T$ for the different seasonal amplitudes noted in the legend. For a given value of $A$, the swelling depth $d$ increases at first as decreases in $M A T$ enable deeper penetration of temperatures cold enough for frost cracking. Near where $M A T=-\Delta T_{c}$, a maximum swelling depth is achieved that is about half the thermal diffusion distance over seasonal periods (i.e. $\frac{1}{2} \sqrt{\kappa \times 6 \text { months }} \approx 2 \mathrm{~m}$ ). Further decreases in MAT are characterized at first by decreases in $d$ as the depth at which the undercooling spends the most time near $\Delta T_{c}$ becomes more shallow (this produces the local maxima in Figure 1 near $5 \mathrm{~m}$ depth for $M A T=-5^{\circ} \mathrm{C}$ and $1 \mathrm{~m}$ depth for $M A T=-10^{\circ} \mathrm{C}$ ). When $M A T$ is sufficiently cold that the undercooling never drops as low as $\Delta T_{c}$, the profiles of $\Delta n(z)$ lose their local maxima (i.e. compare the curves for $A=-5^{\circ}$ and $-10^{\circ} \mathrm{C}$ with the curve for $A=-15^{\circ} \mathrm{C}$ 

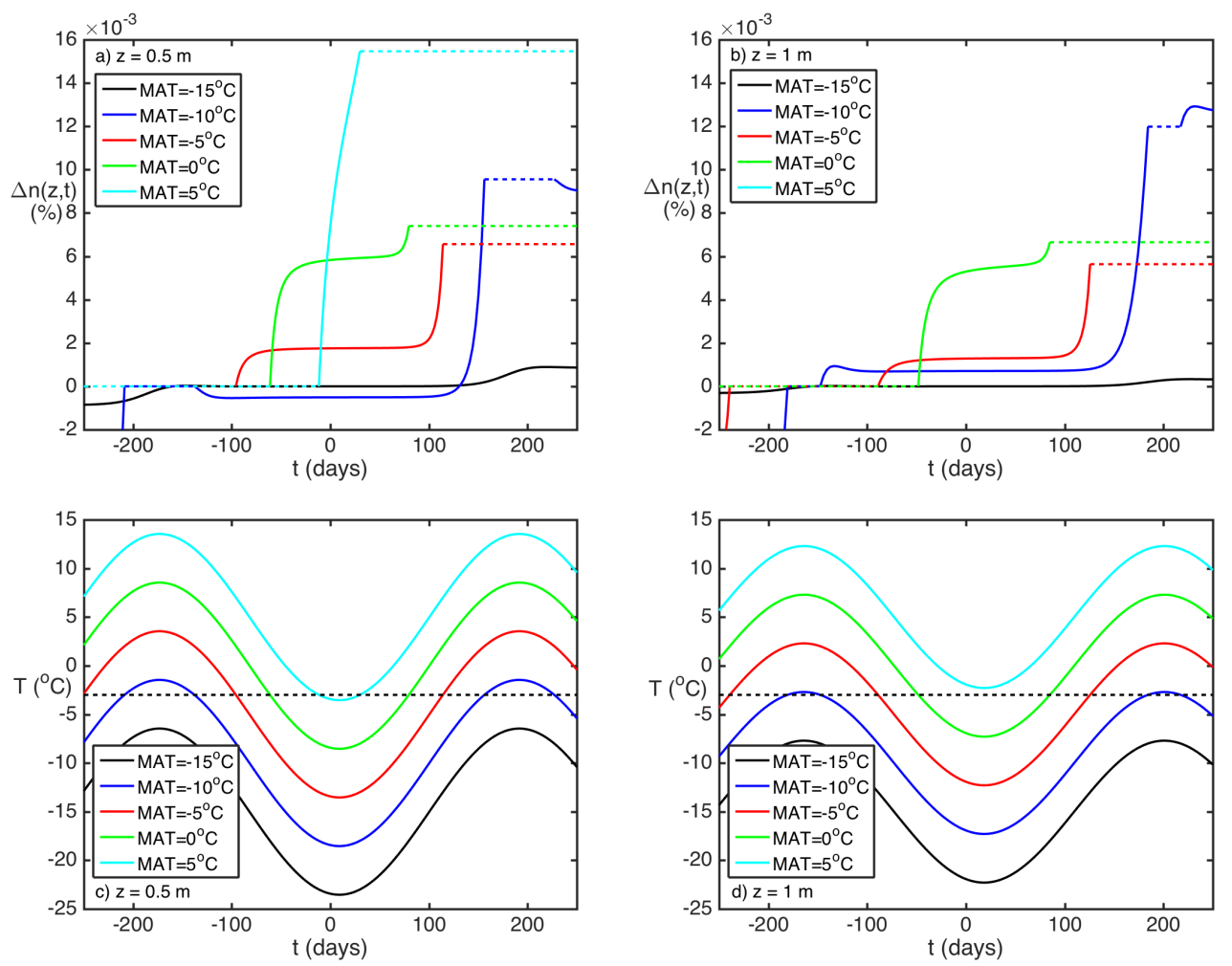

Figure 4: a) and b) Porosity change $\Delta n$ from equation (6), shown as a function of time for the different values of $M A T$ noted in the legends at depth a) $z=0.5 \mathrm{~m}$ and b) $z=1.0 \mathrm{~m}$. c) and d) Temperature histories corresponding with the porosity evolution shown in a) and b). Dashed line indicates the threshold undercooling of $\Delta T_{c}=3{ }^{\circ} \mathrm{C}$. The most rapid increases in $\Delta n$ occur when $T$ is just slightly below $-\Delta T_{c}$.

in Figure 1) and begin to change more slowly with depth as permeability variations become more muted; this causes $d$ to deepen with reduced $M A T$ once again.

The predicted temporal evolution of $\Delta n$ at particular depths provides further insight into the mechanics of frost damage and related geomorphic processes. Figure 4 shows the evolution of porosity at depths of a) $0.5 \mathrm{~m}$ and b) $1.0 \mathrm{~m}$ calculated from equation (6) and corresponding with the history leading up to the plotted points on the graphs shown in Figure 1. Solid portions of the curves trace $\Delta n(z, t)$ through the part of one annual cycle with $\Delta T>\Delta T_{c}$. Warmer time periods during which $\Delta T<\Delta T_{c}$ are marked with horizontal dashed lines. Thermal diffusion delays the minimum temperature at each of these two depths until $t$ is slightly positive, but near the center 
of these plots. When $M A T=5^{\circ} \mathrm{C}$ and $z=0.5 \mathrm{~m}$, the temperature is too warm for frost-cracking to take place for most of the year (except during the month or so spanning $t=0$ ), and at a depth of $z=1.0 \mathrm{~m}$ no frost cracking is expected at all (i.e. the cyan curve in panel d) never dips below the dashed line). At the other end of the range, when $M A T=-15^{\circ} \mathrm{C}$, both depths spend the entire year at undercoolings greater than $\Delta T_{c}$ so frost-cracking can occur, but its rate is limited by extremely low permeabilities at colder temperatures so that the solid black curves in panels a) and b) are nearly horizontal, with slight increases in $\Delta n$ only in the height of summer as $t$ approaches 200 days. Note that cases with $M A T>-\Delta T_{c}$ experience most rapid porosity increases in autumn, whereas the porosity increases more rapidly in spring when $M A T<-\Delta T_{c}$. For the displayed cases where $M A T \geq-5{ }^{\circ} \mathrm{C}$, the porosity evolution is monotonic, with the most rapid changes taking place near the times when the temperature is only slightly colder than the threshold for cracking. When $M A T=-10^{\circ} \mathrm{C}$ the advent of frost cracking in early autumn ( 100 days before midwinter $)$ occurs at a time when the temperature is falling fast enough at $z=0.5 \mathrm{~m}$ that water is first drawn out of the pores (the second term on the right side of equation (6) has a bigger magnitude than the first). This causes the predicted $\Delta n$ to be slightly negative at first (even though $\Delta T>\Delta T_{c}$ ) before experiencing a much larger increase towards the end of the frost-cracking season in the spring. For $M A T=-10^{\circ} \mathrm{C}$ and $z=1.0 \mathrm{~m}$, the frost-cracking season begins a little earlier than when $z=0.5 \mathrm{~m}$, so $\Delta n$ increases slightly before the effects of the second term in equation (6) become important and lead to a slight drop in $\Delta n$ preceding the long period of negligible ice growth before renewed expansion in spring. It is worth emphasizing that the integrated porosity change over an entire frost-cracking season, represented here by the difference between the starting and ending porosity changes along each solid curve, is always positive.

\section{Discussion}

The assumed connection between porosity changes and damage merits further discussion, particularly in light of predictions of small temporary decreases in porosity under certain thermal conditions (e.g. as illustrated in Figure 4 for $M A T=-10^{\circ} \mathrm{C}$ ). It is certainly the case that some porosity change can be reversible; indeed at temperatures warm enough that $\Delta T<\Delta T_{c}$ elastic deformation must accommodate any porosity change since the pressure exerted against pore walls is insufficient to cause fracture propagation. Detailed treatments for the deformation and eventual fracture of idealized pores suggest that more generally the porosity evolution should begin with 
an evolving reversible (elastic) component as pressure builds with decreasing temperature until the onset of a component associated with fracture extension (e.g. Vlahou and Worster, 2010, 2015). Here, it should be recognized that our model treatment is not capable of discriminating between the relative importance of these very different deformation mechanisms; by design, we neither explicitly evolve crack geometry, nor the changes in pore shape facilitated by elastic deformation. However, by tracking only the porosity changes associated with ice growth at temperatures cold enough for cracking, model predictions for $\Delta n$ offer a proxy for the relative degree of frost damage to expect. Section 2 of the Supplementary Information contains a brief comparison of the predictions of the current model against those of several other qualitative treatments for the intensity of frost weathering.

The porosity changes predicted by equations (2) and (3) are completely determined by local conditions; notably, they do not include any dependence on the existence of a $0{ }^{\circ} \mathrm{C}$ reservoir from which to draw liquid. Instead, we assume that sufficient water is present, albeit mostly in a frozen state, that the small porosity changes predicted by our model can be supplied with water from nearby pores. This is one way in which water transport in partially frozen porous media differs fundamentally from the analogous water transport problem in the vadose zone, which is otherwise a very similar physical situation, but is constrained by the meager water content of the vapor phase. In contrast, here since interfacial premelting keeps ice-matrix surfaces wet, the Clapeyron equation (i.e., the description of ice-liquid equilibrium conditions given in Eq. 2 of the Supplementary Information) ensures that liquid availability is maintained as long as ice is present. While we have been explicit in our consideration of only fully-saturated porous media for this model treatment, we have also made the implicit assumption that a water source is available at the ground surface. The annual expansions $\lambda$ that we predict tend to require only modest water additions (i.e. up to a few tenths of a millimeter per year for the conditions represented in Figure 2). If such a water source is unavailable, a modification to this formulation is appropriate in which the first term in the integrand of equation (4) is evaluated beneath the ground surface. Such an extension might be used to examine frost damage under the extremely arid conditions that are present, for example, in the Dry Valleys of West Antarctica and in the Martian regolith, where near-surface pores are typically desiccated (e.g. Hagedorn et al., 2007; Schorghofer and Aharonson, 2005).

Field investigations of contemporary frost damage are challenged by the slow pace of observable change and difficulties with assessing subsurface conditions and behavior. In a novel and logistically 
sophisticated study, Girard et al. (2013) used acoustic emission (AE) data as a proxy for damage on a south-facing Alpine rock wall to infer frost cracking over a broad range of temperatures and moisture contents. At both of their measurement sites, the rate of AE energy detected under freezing conditions was roughly two orders of magnitude larger than under thawed conditions and stayed high to the minimum temperature encountered, near $-15^{\circ} \mathrm{C}$. The absence of a clear low-temperature cutoff to $\mathrm{AE}$ activity is consistent with our model predictions, but our simple formulation also predicts an upper threshold temperature $\left(-\Delta T_{c}\right)$ around which cracking activity is expected to peak (e.g. see Figure 4); no such feature is obvious in the Girard et al. (2013) data set. A key simplification that led to the definition of $\Delta T_{c}$ in equation (1) is that the ice pressure in the region where frost damage takes place is buffered at a characteristic value $P_{c}$, that is dictated by rock properties and crack geometry. With a heterogeneous population of cracks, the critical stress intensity needed to cause failure is expected to scale inversely with the square root of the crack dimensions. This suggests that as cooling progresses, the largest cracks extend first at relatively warm temperatures (i.e. low $\Delta P$ ) that may perhaps even approach $0^{\circ} \mathrm{C}$ for the $\mathrm{cm}-\mathrm{dm}$ scale dimensions detected by Girard et al. (2013). As temperature cools further, the higher ice pressures needed for smaller cracks to extend may eventually be reached. However, premelted pathways should facilitate transport to lower liquid pressures, which would inhibit the growth of smaller cracks. Assuming that the ice pressure in the longest cracks remains fixed at the relatively low levels needed for their propagation, the direct connection between $\Delta P$ and $\Delta T$ implies that the pressure in the premelted liquid lining their walls must stay relatively low as well; this may enable long cracks to continue to grow at cold temperatures by cannibalizing water from their smaller neighbors without necessarily affecting the average porosity in the region. Laboratory observations of frost damage in isothermal systems (e.g. Rempel and Van Alst, 2013) provide evidence for such coarsening behavior. The simplified formulation developed here only accounts for water redistribution that is driven by temperature gradients. A more detailed model that accounts both for the evolution of heterogenous crack populations (i.e. $\left.P_{c}=P_{c}(z, t)\right)$ and unsaturated conditions (possibly generated through cavitation) may shed additional light on the behavior of such systems.

Our model predicts that intense frost damage extends over a broader range of environmental conditions than suggested by previous treatments that emphasize the importance of a liquid-saturated source region (see Section 2 of the Supplementary Information). Evaluation of the swelling depth $d$ suggests that most of this damage should be expected within the upper $2 \mathrm{~m}$ of exposed rock 


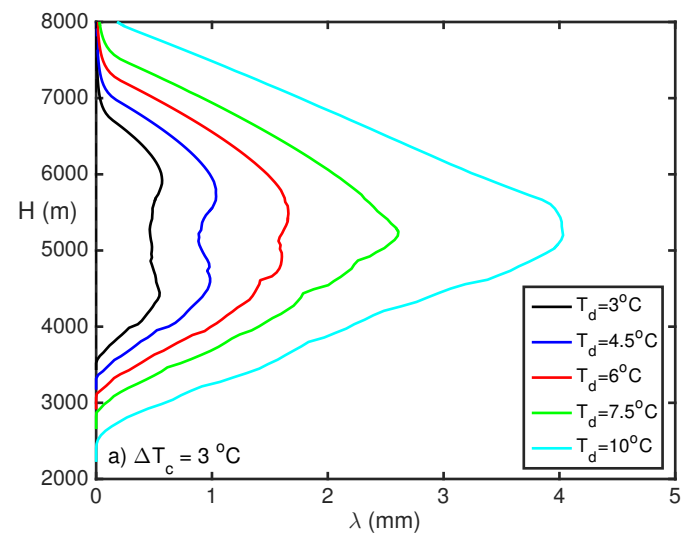
segregation ice growth in the form of lenses.

outcrops. Particularly in deep permafrost conditions, we anticipate intense zones of concentrated damage at depth horizons where the undercooling hovers near $\Delta T_{c}$ for long time periods (e.g. near $1 \mathrm{~m}$ depth when $M A T=-10{ }^{\circ} \mathrm{C}$ for the nominal parameters used to construct Figure 1). This suggests that observations of relict fracture patterns formed in earlier climatic regimes may complement interpretations of other periglacial features (i.e. frost polygons, ice wedges) that have been used to infer past thermal conditions. For example, model reconstructions of the climate during the last glacial maximum suggest that frost-cracking extended over a broad swath of North America spanning south from the Laurentide ice sheet as far as Oregon and Georgia (e.g. French and Millar, 2014; Marshall et al., 2015). We should note that for simplicity, the treatment presented here was designed to treat damage in a homogeneous exposed bedrock. A more detailed model could be constructed to account for surface layers of unconsolidated sediment with the potential to host

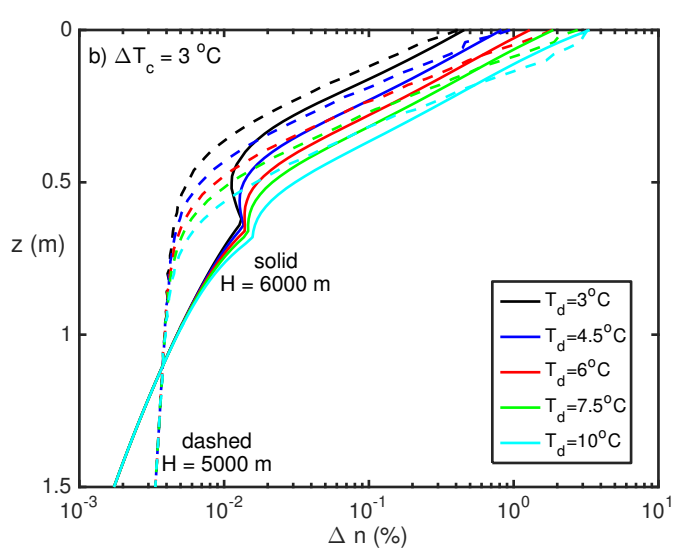

Figure 5: Frost cracking behavior predicted for the Khumbu Himalaya based using an annual sinusoidal temperature signal with $M A T=-20^{\circ} \mathrm{C}$ at $H=8000 \mathrm{~m}$ elevation (lapse rate $-5.8^{\circ} \mathrm{C} / \mathrm{km}$ ) and a halfamplitude of $6.5^{\circ} \mathrm{C}$, as reported by Scherler (2014), and a superimposed sinusoidal diurnal signal with the different amplitudes $T_{d}$ noted in the legends. a) Integrated expansion predicted as a function of elevation (c.f. penalized gradient weighted formulation in Scherler, 2014, Fig. 2E). b) Depth profiles for $\Delta n$ (note logarithmic scale) at $H=5000 \mathrm{~m}$ (dashed) and $6000 \mathrm{~m}$ (solid).

In mountainous terrain, frost-cracking processes have been implicated as a major contributor to head-wall retreat, both within the bergschrunds of cirque glaciers (Sanders et al., 2012), and on unglaciated rock faces (Scherler, 2014; Tucker et al., 2011). Observations of cosmogenic 
nuclides in stream sediments provide further evidence that present-day denudation rates in midlatitude mountain belts are strongly modulated by frost-cracking processes (Delunel et al., 2010; Savi et al., 2015). Our model not only supports these interpretations, which were based upon previous qualitative frost-cracking measures, but also provides further quantitative insight into expected changes in frost damage with elevation. For example, using weather-station data from the Khumbu Himalaya and analysis based upon the penalized-gradient-weighted treatment of Anderson et al. (2013), Scherler (2014) argued that frost-cracking intensity peaks at approximately $6 \mathrm{~km}$ elevation, which coincides with a steepening of ice-free hillslope angles in a number of different lithologies. Using the lapse rate and seasonal temperature amplitude for the current climate, together with the estimated range of diurnal temperature swings reported by Scherler (2014), our model predicts that $\lambda$ is significantly enhanced over a broad range of elevations centered around $5 \mathrm{~km}$ altitude (Figure 5 and Supplementary Figure 3). The different curves in Figure 5a) demonstrate the sensitivity of $\lambda$ to the amplitude of daily temperature excursions that penetrate only to shallow depths. Figure $5 \mathrm{~b})$ compares profiles of $\Delta n(z)$ at elevations of 5 and $6 \mathrm{~km}$ and reveals that the latter exhibit a local maximum at roughly 70 centimeters depth. Given that the predicted magnitudes of $\lambda$ are high at both of these elevations (i.e. see Figure 5a), differences between the character of the predicted profiles of $\Delta n(z)$ may be important for determining the altitudinal dependence of damage that produces significant geomorphic change. For example, if headwall retreat is facilitated by damage to preexisting joints, then peaks in the rate of frost cracking at particular depths would be expected to have profound effects on the weathering rate. Whether or not such a mechanism is important in the Khumbu Himalaya, this example highlights an important consideration in applying any frostcracking model to explain observed geomorphic profiles. Namely that the magnitude of predicted damage is only part of the story, the depth dependence of porosity changes and factors such as the spacing of pre-existing fractures are also important for determining landscape evolution (Hales and Roering, 2009).

The model treatment presented here is designed to incorporate only the simplest set of assumptions necessary to describe how weathering proceeds due to the extension of fractures induced by ice growth. Model validation and refinement would benefit from additional laboratory and field-based constraints that reduce uncertainty in the adopted parameter values, and quantify the timing and degree of frost damage in well-characterized lithologies. Each of the assumptions described in the model development could be relaxed to explore the importance of additional effects. For example, 
complications that might arise from differential growth in a population of cracks has already been mentioned above. The presence of soluble impurities would be expected to modify $T_{m}$ and so affect both the undercooling and permeability at a given temperature. A residual vapor phase component is expected to often be present, and the density change upon solidification undoubtedly can gain importance in certain cases. The model framework presented here can be extended to address each of these complications as circumstances warrant.

\section{Conclusions}

The frost damage that arises from segregation ice growth relies upon the supply of liquid water to growing cracks that are forced open by the pressure ice exerts against crack walls. Pressure gradients in frozen media drive water transport towards colder regions, and the permeability to liquid flow decreases sharply along such flow paths; mass balance requires that porosity increase as a result. In regions cold enough for crack extension, porosity increases are interpreted as a measure of frost damage. Application of our model in an idealized climate with sinusoidally varying surface temperature implies that significant frost damage takes place over a broad range of mean annual temperatures. For the same mean annual temperature and rock properties, damage is expected to be more severe in regions where the seasonal amplitude is larger and correspondingly steeper temperature gradients drive more rapid liquid transport. The reduction in the amplitude of temperature gradients with depth tends to produce a corresponding decrease in the total accumulated frost damage with depth. However, pronounced damage can become localized at particular depth horizons where seasonal variations cause temperatures to linger near the upper limit required for cracking to take place. This upper temperature limit is a consequence of the threshold pressure needed to propagate cracks. No corresponding abrupt lower temperature limit is expected, but the reduction in permeability at colder temperatures can decrease the rate of porosity change to negligible levels. Pore ice can be redistributed and supply the ice growth needed to extend cracks even if the temperature never rises above $0{ }^{\circ} \mathrm{C}$. Spatial and temporal variations in the cracking threshold (due to changes in fracture toughness and/or pore architecture) may produce damage that deviates from the model predictions shown here. 


\section{References}

Akagawa, S., and M. Fukuda (1991), Frost heave mechanism in welded tuff, Permafrost and Periglacial Processes, 2(4), 301-309.

Andersen, J., D. Egholm, M. Knudsen, J. Jansen, and S. Nielsen (2015), The periglacial engine of mountain erosion-part 1: Rates of frost cracking and frost creep, Earth Surface Dynamics Discussions, 3, 285-326.

Andersland, O. B., and B. Ladanyi (2004), Frozen Ground Engineering, John Wiley \& Sons.

Anderson, R. S. (1998), Near-surface thermal profiles in alpine bedrock: Implications for the frost weathering of rock, Arctic and Alpine Research, 30, 362-372.

Anderson, R. S., S. P. Anderson, and G. E. Tucker (2013), Rock damage and regolith transport by frost: An example of climate modulation of the geomorphology of the critical zone, Earth Surface Processes and Landforms, 38(3), 299-316.

Atkinson, B. K. (1984), Subcritical crack growth in geological materials, Journal of Geophysical Research: Solid Earth, 89(B6), 4077-4114.

Clauser, C., and E. Huenges (1995), Thermal conductivity of rocks and minerals, Rock physics 6 phase relations: A handbook of physical constants, pp. 105-126.

Coussy, O. (2005), Poromechanics of freezing materials, Journal of the Mechanics and Physics of Solids, 53(8), 1689-1718.

Dash, J. (1989), Thermomolecular pressure in surface melting: Motivation for frost heave, Science, $246(4937), 1591-1593$.

Dash, J. G., A. W. Rempel, and J. S. Wettlaufer (2006), The physics of premelted ice and its geophysical consequences, Reviews of Modern Physics, 78(3), 695-741.

Davidson, G., and J. Nye (1985), A photoelastic study of ice pressure in rock cracks, Cold Regions Science and Technology, 11(2), 141-153.

Delunel, R., P. A. Van Der Beek, J. Carcaillet, D. L. Bourlès, and P. G. Valla (2010), Frost-cracking control on catchment denudation rates: Insights from in situ produced 10 Be concentrations in 
stream sediments (Ecrins-Pelvoux massif, French Western Alps), Earth and Planetary Science Letters, 293(1), 72-83.

French, H. M. (2013), The Periglacial Environment, John Wiley \& Sons.

French, H. M., and S. W. Millar (2014), Permafrost at the time of the last glacial maximum (LGM) in North America, Boreas, 43(3), 667-677.

Girard, L., S. Gruber, S. Weber, and J. Beutel (2013), Environmental controls of frost cracking revealed through in situ acoustic emission measurements in steep bedrock, Geophysical Research Letters, $40(9)$, 1748-1753.

Hagedorn, B., R. S. Sletten, and B. Hallet (2007), Sublimation and ice condensation in hyperarid soils: Modeling results using field data from Victoria Valley, Antarctica, Journal of Geophysical Research: Earth Surface, 112(F3).

Hales, T., and J. Roering (2009), A frost "buzzsaw" mechanism for erosion of the eastern Southern Alps, New Zealand, Geomorphology, 107(3), 241-253.

Hales, T., and J. J. Roering (2005), Climate-controlled variations in scree production, Southern Alps, New Zealand, Geology, 33(9), 701-704.

Hales, T., and J. J. Roering (2007), Climatic controls on frost cracking and implications for the evolution of bedrock landscapes, Journal of Geophysical Research: Earth Surface, 112(F2).

Hall, K. (2004), Evidence for freeze-thaw events and their implications for rock weathering in northern Canada, Earth Surface Processes and Landforms, 29(1), 43-57.

Hallet, B. (2006), Why do freezing rocks break?, Science, 314(5802), 1092-1093.

Hallet, B., J. Walder, and C. Stubbs (1991), Weathering by segregation ice growth in microcracks at sustained subzero temperatures: Verification from an experimental study using acoustic emissions, Permafrost and Periglacial Processes, 2(4), 283-300.

Ho, E., and W. Gough (2006), Freeze thaw cycles in Toronto, Canada in a changing climate, Theoretical and Applied Climatology, 83(1-4), 203-210. 
Kurylyk, B. L., and K. Watanabe (2013), The mathematical representation of freezing and thawing processes in variably-saturated, non-deformable soils, Advances in Water Resources, 60, 160-177.

Lebeau, M., and J.-M. Konrad (2012), An extension of the capillary and thin film flow model for predicting the hydraulic conductivity of air-free frozen porous media, Water Resources Research, $48(7)$.

Marshall, J. A., J. J. Roering, P. J. Bartlein, D. G. Gavin, D. E. Granger, A. W. Rempel, S. J. Praskievicz, and T. C. Hales (2015), Frost for the trees: Did climate increase erosion in unglaciated landscapes during the late Pleistocene?, Science Advances, 1(10), e1500,715.

McGreevy, J., and W. Whalley (1982), The geomorphic significance of rock temperature variations in cold environments: A discussion, Arctic and Alpine Research, pp. 157-162.

Murton, J. B., R. Peterson, and J.-C. Ozouf (2006), Bedrock fracture by ice segregation in cold regions, Science, $314(5802), 1127-1129$.

Ollier, C., et al. (1984), Weathering., Second edition, Longman Group.

Rempel, A. W. (2010), Frost heave, Journal of Glaciology, 56(200), 1122-1128.

Rempel, A. W., and L. J. Van Alst (2013), Potential gradients produced by pore-space heterogeneities: Application to isothermal frost damage and submarine hydrate anomalies, in Poromechanics V Proceedings of the Fifth Biot Conference on Poromechanics, pp. 813-822, ASCE.

Rempel, A. W., J. S. Wettlaufer, and M. G. Worster (2001), Interfacial premelting and the thermomolecular force: Thermodynamic buoyancy, Physical Review Letters, 87(8), 088,501.

Robertson, E. C. (1988), Thermal properties of rocks, Tech. rep., US Geological Survey,.

Sanders, J. W., K. M. Cuffey, J. R. Moore, K. R. MacGregor, and J. L. Kavanaugh (2012), Periglacial weathering and headwall erosion in cirque glacier bergschrunds, Geology, 40(9), 779 782 .

Savi, S., R. Delunel, and F. Schlunegger (2015), Efficiency of frost-cracking processes through space and time: An example from the eastern Italian Alps, Geomorphology, 232, 248-260.

Scherer, G. W. (1999), Crystallization in pores, Cement and Concrete Research, 29(8), 1347-1358. 
Scherler, D. (2014), Climatic limits to headwall retreat in the Khumbu Himalaya, eastern Nepal, Geology, 42(11), 1019-1022.

Schorghofer, N., and O. Aharonson (2005), Stability and exchange of subsurface ice on Mars, Journal of Geophysical Research: Planets, $110(\mathrm{E} 5)$.

Taber, S. (1930), The mechanics of frost heaving, The Journal of Geology, 38, 303-317.

Tucker, G. E., S. W. McCoy, A. C. Whittaker, G. P. Roberts, S. T. Lancaster, and R. Phillips (2011), Geomorphic significance of postglacial bedrock scarps on normal-fault footwalls, Journal of Geophysical Research: Earth Surface, 116(F1).

Vlahou, I., and M. Worster (2015), Freeze fracturing of elastic porous media: a mathematical model, Proceedings of the Royal Society A, 471.

Vlahou, I., and M. G. Worster (2010), Ice growth in a spherical cavity of a porous medium, Journal of Glaciology, 56(196), 271-277.

Walder, J., and B. Hallet (1985), A theoretical model of the fracture of rock during freezing, Geological Society of America Bulletin, 96(3), 336-346.

Watanabe, K., and M. Flury (2008), Capillary bundle model of hydraulic conductivity for frozen soil, Water Resources Research, $44(12)$.

Wettlaufer, J., and M. G. Worster (2006), Premelting dynamics, Annu. Rev. Fluid Mech., 38, $427-452$ 


\section{Graphical Abstract}
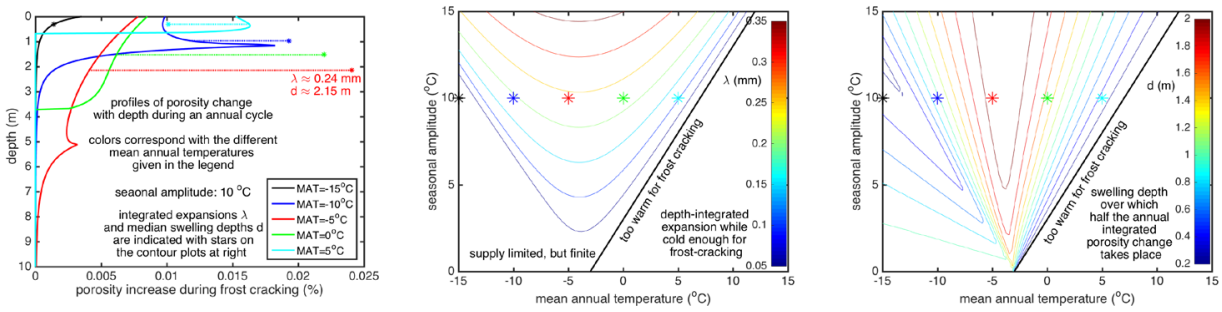